\title{
Prevalencia de disfunción eréctil en hombres del Quindío, y factores de riesgo asociados
}

\section{Prevalence of Erectile Dysfunction in Quindío Men, and Associated Risk Factors}

\author{
Franklin José Espitia De La Hoz ${ }^{1,2,3}$ \\ ${ }^{1}$ Ginecología y Obstetricia, Universidad Militar Nueva Granada, \\ Bogotá, Colombia \\ 2 Uroginecología, FUCS - Hospital de San José, Unicamp, Brasil \\ ${ }^{3}$ Máster en Sexología: Educación y Asesoramiento Sexual,
Address for correspondence Franklin José Espitia De La Hoz, MD, Servicio de Ginecología y Medicina Materno Fetal, Clínica La Sagrada Familia, Armenia, Quindío, Colombia
(e-mail: espitiafranklin@hotmail.com).

Universidad de Alcalá de Henares, Alcalá de Henares, Madrid, Spain

Urol Colomb 2019;28:169-176.

\section{Resumen}

\section{Palabras Clave}

- características culturales

- disfunción eréctil

- enfermedad crónica

- epidemiología

- factores de riesgo
Introducción La disfunción eréctil es la incapacidad persistente, de un hombre, para lograr o mantener una erección que le permita una relación sexual satisfactoria.

Objetivo Conocer la prevalencia de disfunción eréctil en una muestra de hombres del Quindío, y conocer los factores de riesgo relacionados con su aparición.

Materiales y Métodos Estudio descriptivo de corte transversal, en el período de enero de 2010 a diciembre de 2016. Muestra constituida por hombres de 18 a 87 años, los cuales asistieron como acompañantes de las mujeres que acudieron a la Clínica Sagrada Familia de Armenia. La disfunción eréctil se evaluó mediante el cuestionario: Índice Internacional de la Función Eréctil abreviado (IIEF-5).

Resultados Se encuestaron 1.902 hombres, con edad media de 48,6 $\pm 6,9$ años. La media del puntaje en el IIFE-5 fue 17,58 $\pm 5,37$ puntos (rango entre 6 y 25 puntos). La prevalencia de disfunción eréctil en la población general alcanzó un $47,63 \%$, y en la edad o población productiva $39,1 \%$. El $85,81 \%$ de los mayores de 50 años, tenían más disfunción eréctil, comparado con el 15,11\% de los menores de 50 años ( $p<0,0007)$. En cuanto a la intensidad de la disfunción eréctil, la más elevada es la severa $(16,27 \%)$, la moderada $10,46 \%$, y leve a moderada $12,79 \%$. La prevalencia de la severa se incrementó en los mayores de 50 años $(26,64 \%)$ en comparación con los menores de 50 años $(1,92 \%), p=0,0051$. Entre las variables que aumentan el riesgo en este estudio, se destacan: enfermedades crónicas, prostatectomía, tabaquismo, obesidad, sedentarismo, edad superior a 50 años, etc.

Conclusiones Un significativo porcentaje de hombres del Quindío presenta un bajo puntaje en el IIFE-5, compatible con la disfunción eréctil; lo cual está asociado a múltiples factores de riesgo prevalentes en nuestra población.

Introduction Erectile dysfunction is the persistent inability of a man to achieve or maintain an erection that allows him a satisfactory sexual relationship. received

May 25, 2017

accepted

September 29, 2017
DOI https://doi.org/

10.1055/s-0038-1656515.

ISSN 0120-789X.

eISSN 2027-0119.
Copyright $($ C 2019, Sociedad Colombiana License terms de Urología. Publicado por Thieme Revinter Publicações Ltda., Rio de Janeiro, Brazil. Todos los derechos reservados.

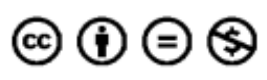




\author{
Keywords \\ - cultural \\ characteristics \\ - erectile dysfunction \\ - chronic disease \\ - epidemiology \\ - risk factors
}

Objective To know the prevalence of erectile dysfunction in a sample of Quindío men, and to know the risk factors related to their appearance.

Materials and Methods Descriptive cross-sectional study, from January 2010 to December 2016. Sample consisting of men aged 18 to 87 years old, who attended as companions of the women who attended the Sagrada Familia Clinic of Armenia. Erectile dysfunction was assessed using the questionnaire: International Index of Abbreviated Erectile Function (IIEF-5).

Results A total of 1902 men were surveyed, with a mean age of $48.6 \pm 6.9$ years. The mean score in the IIFE- 5 was $17.58 \pm 5.37$ points (6-25). The prevalence of erectile dysfunction in the general population reached $47.63 \%$, in the productive $39.1 \%$. $85.81 \%$ of those over 50 years of age had more erectile dysfunction compared with $15.11 \%$ of those younger than 50 years $(p<0.0007)$. As for the intensity of erectile dysfunction, the highest is the severe (16.27\%), the moderate of $10.46 \%$, and mild to moderate, of $12.79 \%$. The prevalence of severe disease increased in those older than 50 years (26.64\%) compared with those younger than 50 years $(1.92 \%), p=0.0051$. Among the variables that increase the risk are: chronic diseases, depression, age over 50 years, history of prostatectomy, etc.

Conclusions A significant percentage of men in Quindío, older than 50 years, presented a low score in IIFE-5, compatible with erectile dysfunction; associated with multiple risk factors prevalent in our population.

\section{Introducción}

La disfunción eréctil es la incapacidad persistente que tiene un hombre, para obtener y mantener una erección, el tiempo necesario, con la suficiente rigidez, que le permita una penetración coital para conseguir un coito satisfactorio hasta el orgasmo y la eyaculación, como mínimo en el 50\% de las relaciones sexuales durante un periodo de tres meses. ${ }^{1,2}$

En múltiples estudios se ha demostrado una relación entre la disfunción eréctil y la hipertensión arterial, ${ }^{3}$ la diabetes mellitus tipo 2 (DM2), ${ }^{4}$ la dislipidemia, ${ }^{5}$ el tabaquismo, ${ }^{6}$ la obesidad, ${ }^{7}$ la depresión, ${ }^{8}$ etc.

Los fenómenos implicados en la disfunción eréctil se explican a través de la microangiopatía y la arteriosclerosis de los vasos, lo que explica la disminución del aporte sanguíneo desencadenando un deficiente aporte de oxígeno en los tejidos, con la consecuente disminución de las concentraciones de óxido nítrico e incremento de la endotelina, manifestándose en la incapacidad para lograr la erección. E igualmente, podrían estar presentes trastornos psicológicos ${ }^{9}$ que preceden o agravan la disfunción.

Se calcula que la prevalencia mundial de disfunción eréctil supera los 100 millones de hombres, y que en los mayores 40 años, alrededor de un 50\%, presenta la disfunción en algún grado de intensidad, con la evidencia de incremento proporcional al aumento de la edad. En los registros de 1995, se menciona que más de 152 millones de hombres, a nivel mundial, habían experimentado disfunción eréctil; y para el 2025, se calculan 322 millones de hombres con la disfunción, representando un incremento de 170 millones. $2,10,11$

El objetivo de esta investigación es conocer la prevalencia de la disfunción eréctil en una muestra de hombres de la población del Quindío, y conocer un aproximado de los factores de riesgo relacionados con su aparición.

\section{Materiales y Métodos}

Estudio descriptivo de corte transversal, en el período comprendido entre el 01 de enero de 2010 y el 31 de diciembre de 2016. La muestra estuvo constituida por hombres de 18 a 87 años, los cuales asistieron como acompañantes de las mujeres que acudieron a la institución por un problema de índole ginecológico, en la Clínica Sagrada Familia, Armenia, Quindío, Colombia, Suramérica.

Los criterios de inclusión consideraron ser hombre de 18 o más años de edad, ser pareja estable y asistir de acompañante de las mujeres que consultaron a ginecología. Se excluyó del estudio los hombres con padecimientos psíquicos o físicos que les dificulten el desarrollo de la actividad sexual coital y los que no deseaban participar en el estudio.

Se les proporcionó un cuestionario a través de una entrevista dirigida para obtener datos socio-demográficos y antecedentes patológicos de las enfermedades asociadas en este estudio. La disfunción eréctil se evaluó mediante un cuestionario estandarizado: Índice Internacional de la Función Eréctil abreviado (IIEF-5). ${ }^{12}$ El cuestionario consta de 5 preguntas con 6 respuestas cada una, con un puntaje que varía de 0 a 5 en cada pregunta ( - Tabla 1). ${ }^{12-14}$ El IIFE-5 en su versión abreviada se enfoca en el diagnóstico de la función eréctil del hombre, en la práctica de la consulta médica cotidiana. El resultado de la puntuación obtenida de cada ítem se suma y se logra un puntaje total. La disfunción se clasifica así: $>=22$ puntos sin disfunción eréctil, con menos de 21 indica disfunción eréctil de diverso grado: 
Tabla 1 Índice Internacional de la Función Eréctil abreviado (IIEF-5)

\begin{tabular}{|c|c|c|c|c|}
\hline \multicolumn{5}{|c|}{ 1. ¿Cómo califica la confianza que tiene en poder mantener una erección? } \\
\hline $\begin{array}{l}1 \\
\text { Muy baja }\end{array}$ & $\begin{array}{l}2 \\
\text { Baja }\end{array}$ & $\begin{array}{l}3 \\
\text { Moderada }\end{array}$ & $\begin{array}{l}4 \\
\text { Alta }\end{array}$ & $\begin{array}{l}5 \\
\text { Muy alta }\end{array}$ \\
\hline \multicolumn{5}{|c|}{$\begin{array}{l}\text { 2. Cuando tiene erecciones mediante estimulación sexual, ¿con qué frecuencia sus erecciones tienen la dureza suficiente para la } \\
\text { penetración? }\end{array}$} \\
\hline $\begin{array}{l}1 \\
\text { Casi nunca }\end{array}$ & $\begin{array}{l}2 \\
\text { Pocas veces }\end{array}$ & $\begin{array}{l}3 \\
\text { Algunas veces }\end{array}$ & $\begin{array}{l}4 \\
\text { Muchas veces }\end{array}$ & $\begin{array}{l}5 \\
\text { Casi siempre }\end{array}$ \\
\hline \multicolumn{5}{|c|}{ 3. Durante las relaciones sexuales, ¿con qué frecuencia puede mantener su erección después de haber penetrado su pareja? } \\
\hline $\begin{array}{l}1 \\
\text { Casi nunca }\end{array}$ & $\begin{array}{l}2 \\
\text { Pocas veces }\end{array}$ & $\begin{array}{l}3 \\
\text { Algunas veces }\end{array}$ & $\begin{array}{l}4 \\
\text { Muchas veces }\end{array}$ & $\begin{array}{l}5 \\
\text { Casi siempre }\end{array}$ \\
\hline \multicolumn{5}{|c|}{ 4. Durante las relaciones sexuales, ¿qué tan difícil es mantener su erección hasta el final del acto sexual? } \\
\hline $\begin{array}{l}1 \\
\text { Extremadamente difícil }\end{array}$ & $\begin{array}{l}2 \\
\text { Muy difícil }\end{array}$ & $\begin{array}{l}3 \\
\text { Difícil }\end{array}$ & $\begin{array}{l}4 \\
\text { Ligeramente difícil }\end{array}$ & $\begin{array}{l}5 \\
\text { Nada difícil }\end{array}$ \\
\hline \multicolumn{5}{|c|}{ 5. Cuando trataba de tener relaciones sexuales, ¿con qué frecuencia eran satisfactorias para usted? } \\
\hline $\begin{array}{l}1 \\
\text { Casi nunca }\end{array}$ & $\begin{array}{l}2 \\
\text { Pocas veces }\end{array}$ & $\begin{array}{l}3 \\
\text { Algunas veces }\end{array}$ & $\begin{array}{l}4 \\
\text { Muchas veces }\end{array}$ & $\begin{array}{l}5 \\
\text { Casi siempre }\end{array}$ \\
\hline \multicolumn{5}{|l|}{ Interpretación de los resultados: } \\
\hline \multicolumn{5}{|c|}{ Sumar los números que corresponden a las respuestas de las preguntas 1 a 5.} \\
\hline \multicolumn{5}{|c|}{$\begin{array}{l}\text { La sumatoria de la puntuación obtenida de todos los ítems, en el IIEF-5, caracteriza la intensidad de la Disfunción Eréctil de la } \\
\text { siguiente manera: }\end{array}$} \\
\hline \multicolumn{5}{|l|}{ 22-25: sin disfunción eréctil } \\
\hline \multicolumn{5}{|l|}{ 17-21: disfunción eréctil leve } \\
\hline \multicolumn{5}{|c|}{ 12-16: disfunción eréctil leve a moderada } \\
\hline \multicolumn{5}{|c|}{$8-11::$ disfunción eréctil moderada } \\
\hline 5-7: disfunción eréctil severa & & & & \\
\hline
\end{tabular}

leve, ${ }^{12,14,15}$ leve a moderada, ${ }^{12,13,16}$ moderada $^{8-11} y$ severa. ${ }^{5-7,12}$

Se consignó además la edad, años de estudio, estado civil, asistencia regular a la iglesia, presencia o no de enfermedades crónicas, cirugía prostática, uso de antihipertensivos, hipoglucemiantes, hipolipemiantes, antecedente de ITS, encuentros sexuales coitales semanales y auto-percepción de salud.

\section{Análisis Estadístico}

Se utilizó el programa Epi-Info versión 6.04 (Centers for Disease Control, USA; OMS, Suiza). La comparación entre los grupos fue evaluada con ANOVA, según homogeneidad de la varianza medida con el test de Bartlett. Los resultados se expresaron como promedio \pm desviación estándar (DS), considerándose significación estadística valores de $p<0,05$. Para la evaluación simultánea de la influencia de diferentes variables sobre la disfunción eréctil se realizó un análisis de regresión logística con el programa Epi-Info 2000.

\section{Resultados}

Se solicitó a 2.160 hombres diligenciar el cuestionario estandarizado: Índice Internacional de la Función Eréctil abreviado (IIEF-5); 258 (11,94\%) rechazaron participar en el estudio. Se presentan los resultados de 1.902 hombres.
Las características biosociodemográficas se muestran en la - Tabla 2. La edad media de todos los encuestados fue de 48,6 \pm 6,9 años; 714 (37,53\%) eran pensionados; 1.449 (76,18\%) eran de religión católica; $1.176(61,82 \%)$ de ellos vivía en unión libre y 726 (38,17\%) eran casados. La frecuencia promedio de actividad sexual era de 3,8 $\pm 1,6$ por mes. La media del puntaje en la escala del IIFE-5 fue de $17,58 \pm 5,37$ puntos (rango entre 6 y 25 puntos). Se observó una escasa proporción de analfabetismo (2,99\%) de predominio en indígenas; en la población general el 38,95\% $(n=741)$ cursaron la primaria, el $51,8 \%(n=987)$ la secundaria y el $9,14 \%(n=174)$ cursaron nivel universitario. No se observaron diferencias sociodemográficas excepto en la raza, entre los hispánicos y el resto. Ésas fueron mayores [65,4\% versus $37,2 \%(p<0,00001)]$.

El 54,1\% (1.029) eran empleados, 8,83\% $(n=168)$ eran usuarios de bloqueadores $\alpha 1$-adrenérgicos para el tratamiento de la hipertrofia prostática benigna; a 375 $(19,71 \%)$ se les había realizado una prostatectomía transuretral, la prostatectomía radical se hizo presente en el $2,83 \%$ ( $n=54)$, y un $94,32 \%$ ( $n=1.794)$ percibían su salud como normal.

El $7,25 \%(n=138)$ fumaba actualmente y el $5,67 \%$ $(n=102)$ fumaba más de 10 cigarrillos al día; sin embargo, se observó un elevado porcentaje de hombres que fumaron en el pasado (27,86\%), de los cuales el $18,76 \%$ 
Tabla 2 Características biosociodemográficas

\begin{tabular}{|l|l|}
\hline Edad media años & $48,6 \pm 6,9(18-87)$ \\
\hline Religión católica & $76,18 \%$ \\
\hline Origen & $67,66 \%(n=1.287)$ \\
\hline Urbana & $32,33 \%(n=615)$ \\
\hline Rural & \multicolumn{2}{|l|}{ Nivel socio-económico } & $6,46 \%(n=123)$ \\
\hline Bajo & $73,59 \%(n=1.398)$ \\
\hline Medio & $20,03 \%(n=381)$ \\
\hline Alto & $62,6 \%(n=1.191)$ \\
\hline Raza & $21,9 \%(n=417)$ \\
\hline Hispánicos & $15,4 \%(n=294)$ \\
\hline Afrocolombianos & $61,82 \%(n=1.176)$ \\
\hline Indígenas & $38,17 \%(n=726)$ \\
\hline Estado civil & $13,74 \pm 3,57$ años \\
\hline Unión libre & $37,53 \%(n=726)$ \\
\hline Casados & $54,1 \%(n=1.029)$ \\
\hline Tiempo de convivencia & $16,8 \pm 1,98$ \\
\hline Ocupación & $3.8 \pm 1.6$ mensual \\
\hline Pensionados & $17,58 \pm 5,37(6-25)$ \\
\hline Empleados & $94,32 \%(n=1.794)$ \\
\hline Inicio de la vida sexual \\
(años de edad)
\end{tabular}

Abreviaciones: IIFE-5, índice internacional de la función eréctil abreviado.

Tabla 3 Prevalencia de enfermedades crónicas y condiciones de riesgo en hombres con disfunción eréctil en el Quindío

\begin{tabular}{|l|l|}
\hline Edad $>50$ años & $45,11 \%(n=858)$ \\
\hline HTA & $18,29 \%(n=348)$ \\
\hline Diabetes & $9,14 \%(n=174)$ \\
\hline Dislipidemia & $11,98 \%(228)$ \\
\hline Depresión & $4,5 \%(n=87)$ \\
\hline Hipotiroidismo & $4,4 \%(n=84)$ \\
\hline IMC media & $29,4 \pm 3,9(21,6-48)$ \\
\hline Ingesta de alcohol & $85,64 \%(n=1.629)$ \\
\hline Fumadores & $7,25 \%(n=138)$ \\
\hline Prostatectomía transuretral & $19,71 \%(n=375)$ \\
\hline Prostatectomía radical & $2,83 \%(n=54)$ \\
\hline ITS & $6,15 \%(n=117)$ \\
\hline Sedentarismo & $61,67 \%(n=1.173)$ \\
\hline
\end{tabular}

Abreviaciones: HTA, hipertensión arterial; IIFE-5, índice internacional de la función eréctil abreviado; IMC, índice de masa corporal; ITS, infecciones de trsmisión sexual. lo abandonó cinco años antes del estudio. El 14,35\% ( $n=273)$ no consumía alcohol en ninguna circunstancia y el 21,6\% $(n=411)$ ingería más de 30 gramos de alcohol al día (3 o más copas de vino). El 32,64\% (621) no padecía ninguna enfermedad crónica.

En relación al sedentarismo se encontró que el 61,67\% ( $n=1.173$ ) de los hombres del estudio, pasaba más de seis horas al día sentado, siendo el porcentaje mayor en los pensionados (67,76\%) que en los empleados (44,48\%); y alrededor de la cuarta parte de los hombres (24,45\%) realizaba semanalmente actividad física en los tiempos recomendados por su médico tratante.

Los universitarios fueron los más delgados, y mostraron menor prevalencia de dislipidemia $(11,7 \%)$, sin diferencias en relación con el colesterol LDL $>100$. Los hispánicos mostraron disminución del IMC frente a los no hispánicos [24,9 versus 28,2 , respectivamente, $(p=0,01)]$ y una probabilidad inferior al $48 \%$ de presentar sobrepeso $\mathrm{y}$ obesidad.

El nivel de educación fue inferior en mayores de 50 años con un puntaje significativamente menor en el IIEF-5, que en los menores de 50 años; también se encontraron diferencias en cuanto al consumo de alcohol, con un menor puntaje en el IIEF-5 $(p<0,001)$ y una frecuencia superior de disfunción severa $(p<0,001)$. El porcentaje de disfunción eréctil observado en los indígenas también fue claramente inferior en los analfabetos ( $p<0,001)$.

$\mathrm{Al}$ analizar el estado civil, la religión y el antecedente de ITS, no se encontró ningún efecto relacionado con reducción en el puntaje del IIEF-5, o simplemente no fue significativo $(p=0,087)$.

En la - Tabla 3 se describen las enfermedades crónicas y las condiciones de salubridad, con mayor prevalencia, en los hombres evaluados en esta investigacion por disfunción eréctil.

La hipertensión arterial mostró una prevalencia del $18,29 \%(n=348)$, la diabetes mellitus el 9,14\% $(n=174)$, la dislipidemia el $11,98 \%(n=228)$, la depresión el $4,5 \%$ $(n=87)$, el hipotiroidismo el $4,4 \%(n=84)$. La diabetes mellitus tipo 2, fue la enfermedad endocrina más asociada a la hipertensión arterial. Todos los hombres estaban en control de su condición clínica, perteneciendo al grupo de "pacientes crónicos."

La media de los años de evolución de la hipertensión arterial fue de 17.34 años (rango entre 1 y 42 años). Entre el grupo de hipertensos el 48,27\% $(n=168)$ tenía una evolución mayor de 10 años. Los que seguían un tratamiento exclusivamente con medidas higiénicodietéticas, fueron el grupo más bajo con el $4,88 \%$.

Entre los hombres hipertensos el $42,24 \%$ ( $n=147$ ) usaba la asociación de dos o más fármacos antihipertensivos. El grupo farmacológico más utilizado fue el de los antagonistas de los receptores de la angiotensina II (ARA II), presente en el 55,17\% $(n=192)$ de los hombres hipertensos participantes en el estudio, seguidos de los $\beta$-bloqueantes adrenérgicos y los calcio-antagonistas. La estatina más usada era la lovastatina, y la metformina entre los hipoglucemiantes orales.

El 79,31\% ( $n=1.044)$ de los hombres menores de 50 años mantenían relaciones sexuales coitales dos veces por 
semana, frente a un $52,79 \%(n=453)$ de los mayores de 50 años $(p<0,0001)$. El grupo que reportó mayor frecuencia de encuentros sexuales coitales fue el de 18-35 años, con $5,4 \pm 2,7$ (4-14) encuentros semanales. Número que disminuye de forma progresiva y significativa hasta $1,2 \pm 1,8$ (1-5) de encuentros mensuales en los mayores de 75 años $(p<0,0002)$; demostrando que la edad se correlaciona con la frecuencia del número de encuentros sexuales coitales, por lo tanto, son más frecuentes en los hombres más jóvenes $(p<0,0001)$.

En relación con los hombres del área urbana y los del área rural, se observó una mayor frecuencia y preocupación por una vida sexual satisfactoria entre los hombres de la ciudad, siendo significativamente mayor con el $81,35 \%$ y el $59,51 \%$, respectivamente $(p=0,005)$.

El hecho de ser profesionales o desempeñarse en determinada ocupación laboral no se relacionó con las diferencias significativas en la presencia de disfunción eréctil $(p=0,07)$, aunque la frecuencia de encuentros sexuales coitales semanales fue significativamente mayor en los que desempeñaban una actividad laboral independiente, sin patrón o jefe inmediato, 3,9 $\pm 2,1$ $(p=0,003)$.

En los datos ajustados para la población estudiada, las proporciones de hombres con diabetes $(p<0,001)$, hipertensión arterial $(p<0,001)$, dislipidemia $(p=0,01)$ y obesidad $(p<0,001)$ fueron significativamente más altas en la población de mayores de 50 años que en la de menores de 50 años. Hubo también leves diferencias no significativas en la prevalencia de hipotiroidismo y depresión.

En la - Tabla 4 se muestra la prevalencia e intensidad de la disfunción eréctil de acuerdo a grupos etarios de los hombres agrupados por edad. Se observa en ellos una prevalencia significativa en el grupo de más de 75 años $(n=258)$ cuyo porcentaje del 39,53\% ( $n=102)$, comparada con los más jóvenes (menores de 35 años), de 5,84\% $(n=27)$ $p<0,0001$ ). Los hombres sin práctica de actividad física tuvieron menor puntuación en el IIEF-5, al compararlos con los que si la realizaban $(6,9 \pm 2,1$ versus $8,1 \pm 1,5, p<0,04$, respectivamente). Se encontró una correlación negativa entre el tabaquismo y la puntuación del IIEF-5 $(p<0,042)$. En cuanto a la intensidad evaluada por el IIFE-5, la más elevada es la severa (16,27\%) en el mismo grupo de más de 75 años, que se incrementa desde $0,64 \%(n=3)$ de los más jóvenes ( $p<0,0001$ ); la moderada de $10,46 \%$, desde $1,21 \%$ $(p<0,001)$ y leve a moderada, de $12,79 \%$ desde $2,59 \%$ $(p<0,001)$. Se observa que la puntuación total del IIFE-5
Tabla 5 Factores de riesgo para disfunción eréctil en hombres del Quindío

\begin{tabular}{|l|l|l|l|}
\hline & OR & IC 95\% & Valor $\mathbf{p}$ \\
\hline Enfermedades crónicas & 3,75 & $1,26-12,9$ & 0,003 \\
\hline Analfabetismo & 3,57 & $1,35-9,24$ & 0,003 \\
\hline Depresión & 3,48 & $1,47-8,43$ & 0,003 \\
\hline Edad >50 años & 2,79 & $1,17-3,27$ & 0,005 \\
\hline Prostatectomía & 2,67 & $1,35-3,81$ & 0,005 \\
\hline Población rural & 2,04 & $1,14-3,54$ & 0,001 \\
\hline Nivel socio-económico bajo & 1,98 & $1,05-3,18$ & 0,002 \\
\hline IMC & 1,95 & $0,57-6,06$ & 0,004 \\
\hline Ingesta de alcohol & 1,83 & $1,02-3,26$ & 0,003 \\
\hline Sedentarismo & 1,76 & $1,02-1,95$ & 0,033 \\
\hline Tabaquismo & 1,74 & $1,11-3,36$ & 0,008 \\
\hline Antidepresivos & 1,71 & $1,05-1,92$ & 0,036 \\
\hline Hipoglucemiantes & 1,26 & $2,37-7,62$ & 0,025 \\
\hline Antihipertensivos & 0,93 & $0,3-5,61$ & 0,037 \\
\hline Hipolipemiantes & 0,81 & $1,02-6,06$ & 0,048 \\
\hline $\begin{array}{l}\text { Percepción de su } \\
\text { salud normal }\end{array}$ & 0,72 & $0,42-1,08$ & 0,153 \\
\hline
\end{tabular}

Abreviaciones: IC, intervalo de confianza; IMC, índice de masa corporal; OR, odds ratio.

menor de 22, aumenta significativamente con la edad; la mayoría $(85,81 \%)$ de los hombres mayores de 50 años, tenían más disfunción eréctil, comparado con el $15,11 \%$ de los encuestados menores de 50 años $(p<0,0007)$.

En la - Tabla 5 se observa la influencia de múltiples variables que afectan de forma negativa sobre el riesgo de presentar una disfunción eréctil. Entre las variables que aumentan el riesgo significativamente, se destacan las enfermedades crónicas, la depresión, la edad superior a 50 años, el antecedente de prostatectomía, el origen rural, el nivel socio-económico bajo, la obesidad, la ingesta de alcohol, el sedentarismo, el tabaquismo, así como los medicamentos para las terapias crónicas. La percepción de su salud normal fue un factor que tendió a ser protector.

La prevalencia de disfunción eréctil severa se incrementó significativamente en los hombres mayores de 50 años de edad $(26,64 \%)$ en comparación con los hombres de menos de 50 años $(1,92 \%), p=0,0051$. Entre los factores que se relacionan con la aparición de ese grado de disfunción, se encontraron la

Tabla 4 Prevalencia e intensidad de la disfunción eréctil en hombres del Quindío

\begin{tabular}{|l|l|l|l|l|l|l|}
\hline Edad años & Población & Prevalencia & Leve & Leve a moderada & Moderada & Severa \\
\hline $18-35$ & 462 & $5,84 \%$ & $2,07 \%$ & $2,16 \%$ & $1,08 \%$ & $0,36 \%$ \\
\hline $36-50$ & 582 & $9,27 \%$ & $3,42 \%$ & $3,12 \%$ & $1,17 \%$ & $1,56 \%$ \\
\hline $51-65$ & 351 & $21,36 \%$ & $5,70 \%$ & $4,83 \%$ & $4,92 \%$ & $5,73 \%$ \\
\hline $66-75$ & 303 & $24,75 \%$ & $4,30 \%$ & $5,64 \%$ & $6,45 \%$ & $8,07 \%$ \\
\hline$>75$ & 204 & $39,70 \%$ & $5,38 \%$ & $10,59 \%$ & $10,71 \%$ & $12,84 \%$ \\
\hline
\end{tabular}


hipertensión, la prostatectomía y la diabetes, así como el uso de medicamentos para tratar esas enfermedades.

Se evaluaron las diferencias de la prevalencia de disfunción eréctil entre los hombres diabéticos que tenían menos y más de 10 años de diagnóstico; esos últimos fueron mayores $(p<0,0001)$, y las prevalencias de hipertensión arterial y de IMC aumentado fueron superiores $(p=0,01 \mathrm{y}$ $p=0,003$, respectivamente), sin mayores diferencias con las otras variables.

La correlación que existe entre la presencia de dos o más comorbilidades con el puntaje IIFE- 5 resultó negativa con significancia estadística $(p=0,0021)$, observándose que un menor puntaje traduce un mayor grado de disfunción eréctil.

La prevalencia de disfunción eréctil se incrementó con el aumento de la edad (seis veces más alta entre las edades de más de 50 años que entre los menores de 35 años); y 1,8 veces mayor en los diabéticos respecto a los no diabéticos $(p=0.0027)$.

Al final del estudio se demostró que la prevalencia de disfunción eréctil, con algún grado de intensidad en la población general, fue del $47,63 \%(n=902)$, y los factores de riesgo asociados con más prevalencia a la disfunción son: la depresión, la edad mayor a 50 años, residir en población rural, las enfermedades crónicas, el antecedente de prostatectomía, el sedentarismo, el analfabetismo, el tabaquismo, la obesidad, pertenecer al nivel socioeconómico bajo y la ingesta de alcohol.

\section{Discusión}

Es curioso el elevado interés de los hombres en participar en la investigación, aun después de observar las preguntas del cuestionario, tal vez, alentados porque se les aseguró la confidencialidad del mismo. En eso podría influir la idiosincrasia paisa por su espíritu proactivo y emprendedor, lo que explicaría la motivación y el deseo de conocer su situación sexual.

Siguiendo que la Presidencia de la República sancionó la Ley 1821 del 30 de diciembre de 2016 con la cual se amplía a 70 años la edad máxima para desempeñar funciones públicas en el Estado; en este estudio la población productiva se extendió hasta los 70 años por lo que, la prevalencia estimada de disfunción eréctil se estableció entre hombres de 18 y 70 años $(n=1.395)$, la cual fue del $39,13 \%$ ( $n=546)$; cifra menor a la registrada por otros estudios en que se reportó entre un $53,4 \%$ a un $88 \%^{4,13}$

En los hombres con Diabetes Mellitus, la prevalencia de disfunción eréctil en el presente estudio, fue de un 65,51\%, muy superior al $35 \%{ }^{15}$ y $40 \%{ }^{16}$ reportado por otros autores.

En la presente investigación, los hombres con dos o más enfermedades crónicas muestran una mayor prevalencia disfunción eréctil, encontrándose asociación estadística con la hipertensión, la diabetes mellitus y la cardiopatía isquémica; resultando claro que son múltiples las variables asociadas al trastorno, principalmente, las relacionadas con las enfermedades cardiovasculares $\mathrm{y}$ las alteraciones metabólicas, tal como lo describen otros estudios. ${ }^{17,18}$

La percepción de los hombres participantes en este estudio, es que la disfunción eréctil se exacerbó luego del inicio del tratamiento farmacológico, especialmente entre los hipertensos, lo que es concordante con otras publicaciones; ${ }^{19,20}$ siendo el carvedilol el medicamento relacionado con mayor empeoramiento de la disfunción eréctil, reafirmando lo descrito por anteriores estudios. ${ }^{21,22}$ El valsartán demostró proporcionar mejores beneficios entre los antihipertensivos, ya que los hombres no se quejaron de agravarse su disfunción una vez iniciada la terapia, e incluso resultó curioso observar que algunos hombres manifestaron experimentar un aumento (1-3 encuentros más al mes) en la frecuencia de las relaciones sexuales.

La depresión se encontró en el 68,8\% de los hombres con disfunción eréctil severa, constituyéndose en un factor a tener en cuenta, además de observarse una mayor prevalencia de disfunción eréctil entre los hombres tratados con psicofármacos (87,15\%), cifra superior a lo reportado por otros autores. ${ }^{23,24}$

El 39,64\% de los hombres casados, de más de 60 años de edad, presentaban disfunción eréctil en algún grado de intensidad, resultado superior al registrado en la literatura; ${ }^{25}$ además de observarse una mayor disfunción eréctil en los que llevaban más de 10 años en pareja.

Entre los hombres con hiperplasia prostática benigna (41,64\%), se encontró disfunción eréctil en el 57,95\% $(n=495)$ de los encuestados, porcentaje elevado en relación a otras publicaciones (30\% a 33\%), 26,27 elevándose la cifra al $78,4 \%$ en el grupo de los prostatectomizados, siendo el $100 \%$ en los que se les realizó prostatectomía radical, acorde a lo publicado por otros estudios. ${ }^{28,29}$

En esta investigación, el IIEF-5 demostró ser una herramienta fácil de usar, igual de reproducible y fiable en cualquier grupo de hombres para el diagnóstico de la disfunción eréctil, tal como ha sido descrito. ${ }^{4-6,12}$

Es llamativa la alta frecuencia de sensación de alteración en la masculinidad percibida por los hombres, a medida que se agrava la intensidad de la disfunción eréctil, lo cual puede indicar que la erección sigue siendo la función relevante, entre esos hombres, a la hora de intimar, tal como ha sido descrito previamente en otras publicaciones. ${ }^{30,31}$

La significativa diferencia observada en la prevalencia de la disfunción eréctil entre la población general, la población productiva y los hombres mayores de 50 años, se explica por la presencia de dos o más factores de riesgo en la población en general, sin contar con adecuados hábitos alimenticios ni rutina de ejercicio regular, mientras en la población productiva la obesidad se destacaba asociada al tabaquismo, ingesta de alcohol y sedentarismo, los cuales constituyen un coctel negativo para la erección, sin embargo, en los mayores de 50 años, tenían más de tres factores de riesgo asociados a la mayor edad, demostrando que el envejecimiento, independientemente de las comorbilidades, es un importante factor de riesgo para el desarrollo de la disfunción eréctil en los hombres, en lo cual, este estudio muestra concordancia con otras publicaciones. ${ }^{32-34}$ Otra razón para las disparidades existentes en la prevalencia de esos grupos, se debe a diferencias en la composición de la población del estudio.

En relación a lo multicausal y multidimensional de la disfunción eréctil, es importante considerar en próximas 
investigaciones, otros factores relacionados con su aparición como el contexto de la pareja, los aspectos psicológicos y el ambiente socio-cultural del individuo.

\section{Limitaciones}

En las limitaciones de esta investigación se destacan el uso del IIFE-5, que no está validado para la población colombiana, sin que ello le reste valor a su reconocida aplicabilidad. Lo otro se relaciona con la muestra obtenida de forma consecutiva, sabiendo los impedimentos de generalización que eso supone. No obstante, a pesar de todo ello, se logró el objetivo del estudio.

\section{Conclusiones}

La prevalencia de disfunción eréctil en la población del Quindío es alta entre los hombres con dos o más factores de riesgo, y mayor en los sometidos a la prostatectomía radical.

La prevalencia de disfunción eréctil severa es superior en los mayores de 50 años, constituyéndose la edad en una amenaza para los hombres.

Las enfermedades crónicas, la depresión, el analfabetismo, el origen rural, el nivel socio-económico bajo, la obesidad, la ingesta de alcohol, el sedentarismo, el tabaquismo y los medicamentos para las terapias crónicas fueron predictores significativos de disfunción eréctil.

\section{Responsabilidades Éticas}

Protección de personas y animales. Los autores declaran que los procedimientos seguidos se conformaron a las normas éticas del comité de experimentación humana responsable y de acuerdo con la Asociación Médica Mundial y la Declaración de Helsinki.

Confidencialidad de los datos. Los autores declaran que han seguido los protocolos de su centro de trabajo sobre la publicación de datos de pacientes.

Derecho a la privacidad y consentimiento informado. Los autores han obtenido el consentimiento informado de los pacientes y/o sujetos referidos en el artículo. Este documento obra en poder del autor de correspondencia.

Conflictos de Interés

Los autores declaran no tener conflictos de interés.

\section{Financiamiento}

Los autores no han recibido ayuda financiera de ninguna entidad, el resultado de este estudio ha sido logrado gracias al esfuerzo económico del autor.

\section{Agradecimientos}

A la doctora Lilian Orozco Santiago por esmerarse en la búsqueda de la bibliografía relacionada con la investigación. Agradecemos a La Clínica La Sagrada Familia por su ayuda en la impresión de los cuestionarios, y a todas las enfermeras que nos apoyaron por su esmerado trabajo; sin su valioso aporte este estudio no se hubiese realizado.

\section{Bibliografía}

1 Korfage IJ, Pluijm S, Roobol M, Dohle GR, Schröder FH, Essink-Bot ML. Erectile dysfunction and mental health in a general population of older men. J Sex Med 2009;6(02):505-512

2 NIH Consensus Development Panel on Impotence. NIH Consensus Conference. Impotence. JAMA 1993;270(01):83-90

3 Virag R, Bouilly P, Frydman D. Is impotence an arterial disorder? A study of arterial risk factors in 440 impotent men. Lancet 1985;1 (8422):181-184

4 Benet AE, Melman A. The epidemiology of erectile dysfunction. Urol Clin North Am 1995;22(04):699-709

5 Seftel AD, Sun P, Swindle R. The prevalence of hypertension, hyperlipidemia, diabetes mellitus and depression in men with erectile dysfunction. J Urol 2004;171(6 Pt 1):2341-2345

6 Tengs TO, Osgood ND. The link between smoking and impotence: two decades of evidence. Prev Med 2001;32(06):447-452

7 Derby CA, Mohr BA, Goldstein I, Feldman HA, Johannes CB, McKinlay JB. Modifiable risk factors and erectile dysfunction: can lifestyle changes modify risk? Urology 2000;56(02): 302-306

8 Araujo AB, Durante R, Feldman HA, Goldstein I, McKinlay JB. The relationship between depressive symptoms and male erectile dysfunction: cross-sectional results from the Massachusetts Male Aging Study. Psychosom Med 1998;60 (04):458-465

9 Roth A, Kalter-Leibovici O, Kerbis Y, et al. Prevalence and risk factors for erectile dysfunction in men with diabetes, hypertension, or both diseases: a community survey among 1,412 Israeli men. Clin Cardiol 2003;26(01):25-30

10 Laumann EO, Nicolosi A, Glasser DB, et al; GSSAB Investigators' Group. Sexual problems among women and men aged 40-80 y: prevalence and correlates identified in the Global Study of Sexual Attitudes and Behaviors. Int J Impot Res 2005;17(01): 39-57

11 Feldman HA, Goldstein I, Hatzichristou DG, Krane RJ, McKinlay JB. Construction of a surrogate variable for impotence in the Massachusetts Male Aging Study. J Clin Epidemiol 1994;47(05): 457-467

12 Rosen RC, Cappelleri JC, Smith MD, Lipsky J, Peña BM. Development and evaluation of an abridged, 5-item version of the International Index of Erectile Function (IIEF-5) as a diagnostic tool for erectile dysfunction. Int J Impot Res 1999;11 (06):319-326

13 Cappelleri JC, Siegel RL, Osterloh IH, Rosen RC. Relationship between patient self-assessment of erectile function and the erectile function domain of the international index of erectile function. Urology 2000;56(03):477-481

14 Karakiewicz P, Shariat SF, Naderi A, Kadmon D, Slawin KM. Reliability of remembered International Index of Erectile Function domain scores in men with localized prostate cancer. Urology 2005;65(01):131-135

15 McCulloch DK, Campbell IW, Wu FC, Prescott RJ, Clarke BF. The prevalence of diabetic impotence. Diabetologia 1980;18(04): 279-283

16 González-Juanatey JR, Alegría Ezquerra E, Gomis Barberá R, et al; Estudio DIVA. Disfunción eréctil como marcador de vasculopatía en la diabetes mellitus tipo 2 en España. Estudio DIVA. Med Clin (Barc) 2009;132(08):291-297

17 Jensen J, Lendorf A, Stimpel H, Frost J, Ibsen H, Rosenkilde P. The prevalence and etiology of impotence in 101 male hypertensive outpatients. Am J Hypertens 1999;12(03):271275

18 Greenstein A, Chen J, Miller H, Matzkin H, Villa Y, Braf Z. Does severity of ischemic coronary disease correlate with erectile function? Int J Impot Res 1997;9(03):123-126

19 Greenberg G, Brennan PJ, Miall WE. Effects of diuretic and betablocker therapy in the Medical Research Council Trial. Am J Med 1984;76(2A):45-51 
20 Rosen RC. Sexual dysfunction as an obstacle to compliance with antihypertensive therapy. Blood Press Suppl 1997;1(01):47-51

21 Cordero A, Bertomeu-Martínez V, Mazón P, et al. Erectile dysfunction in high-risk hypertensive patients treated with beta-blockade agents. Cardiovasc Ther 2010;28(01):15-22

22 Shabsigh R, Perelman MA, Lockhart DC, Lue TF, Broderick GA. Health issues of men: prevalence and correlates of erectile dysfunction. J Urol 2005;174(02):662-667

23 Segraves RT, Balon R. Antidepressant-induced sexual dysfunction in men. Pharmacol Biochem Behav 2014;121:132-137

24 Werneke U, Northey S, Bhugra D. Antidepressants and sexual dysfunction. Acta Psychiatr Scand 2006;114(06):384-397

25 Diokno AC, Brown MB, Herzog AR. Sexual function in the elderly. Arch Intern Med 1990;150(01):197-200

26 Steg A, Zerbib M, Conquy S. [Sexual disorders after an operation for benign prostatic hypertrophy]. Ann Urol (Paris) 1988;22(02): 129-133

27 Amat Cecilia M, Romero Pérez P, Omera-Arbash AR. Actividad sexual tras la cirugía de la hipertrofia prostática benigna. Arch Esp Urol 1993;46(08):683-687, discussion 687-688
28 Catalona WJ, Bigg SW. Nerve-sparing radical prostatectomy: evaluation of results after 250 patients. J Urol 1990;143(03): 538-543, discussion 544

29 Walsh PC, Donker PJ. Impotence following radical prostatectomy: insight into etiology and prevention. J Urol 1982;128(03): 492-497

30 Espitia De La, Hoz FJ. FJ. Sexo Ilimitado/El vuelo hacia una sexualidad más placentera. Primera edición. Bogotá. Editorial Bolívar 2015;5(02):247-248

31 Espitia De La, Hoz FJ. Mi primer año en La Crónica. Primera edición. Bogotá. Editorial Bolívar 2016:150-151

32 Weber MF, Smith DP, O'Connell DL, et al. Risk factors for erectile dysfunction in a cohort of 108477 Australian men. Med J Aust 2013;199(02):107-111

33 Cheng JY, Ng EM, Chen RY, Ko JS. Prevalence of erectile dysfunction in Asian populations: a meta-analysis. Int J Impot Res 2007;19(03):229-244

34 Bai Q Xu QQ, Jiang H, Zhang WL, Wang XH, Zhu JC. Prevalence and risk factors of erectile dysfunction in three cities of China: a community-based study. Asian J Androl 2004;6(04):343-348 